\title{
应用声笔数字化器一一计算机分析 超声心动图的初步研究
}

\author{
张鸣晓 马星暯 肖 平 \\ (北京同上医院内科) \\ 胡广书院建国 周礼某 \\ (清华大学生物医学电子工程研究室)
}

近年来应用超声心动图 (Echocardiogram 以下简称 UCG) 作为一种无创伤沴断心肚疾病 的新技术，被人们公认为诊断不可缺少的重要手段之一. 由于目前我国多采用手工方法测量 UCG,不仅效率低，甚至丟失了不少有意义的信息，影响了 UCG 在科研和实际应用中的价值. 我们采用由声笔数字化器和计算机组成的 UCG 自动分析系统, 不仅提高 UCG 分析计算效 率,而且解决了手工方法难以得到的许多数据. 为检验本系统的功能、准确性, 可靠性和实用 价值, 选择 97 例健康成人进行 UCG 检查, 与常规用尺测量加计算器计算后所得结果相比较, 现分述如下:

\section{一、一般资料、仪器和方法}

1. 从九个单位、三万名职工中, 经过多年临床系统观察、体检、胸透及心电图检查均風正 常, 并详细询问个人及家族史中均无心血管系统及能影响心血管系统的其它疾病者共 97 人. 年龄 19-59 岁,男性 55 名、女性 42 名.

2. 应用 Aloka SSD- $110 \mathrm{~S}$ 型超声心动图仪. 受检者休息 15 分钟后, 平卧位及 30 度方侧 卧位,探头置于胸骨左缘第 3 或 4 肋间, 当探测到准确的 4、3、2 区曲线后, 用 135 照相机摄影 记录,将图象清晰的胶卷供测量并计算左心室功能。

3. 整个系统由美国 (Cromemco Z80) 微型计算机和美国科学附件公司 (Science Acressories Corporation 简称 SAC)生产的 Graf/pen 声笔数字化器(Sonic pen digitizer)组成,后者包括声笔控 制器、L 型直角坐标传感器和声笔, 其功能是将 L 型传感器 $35 \times 35 \mathrm{~cm}^{2}$ 有效范围内“点”的坐 标转换成一对数字量，并按一定的方式送人计算机。使用时先把 L 型传感器固定于平板上,其 下安装一台放大机, 将被测的 UCG135 底片放置在传感器有效范围内的平板上. 根据声管数 字化器的原理, 设计了一种“点菜式”的操作方式, 即把要测量的 60 个数据和 9 条命令, 构成一 个被称为“菜单”的表格, 置于测量平板的右下角, 当声笔触及表上任何一格时,计算机根据这 一点的坐标，便可感受操作者“点”的是哪一种“莱”而执行相应的计算. 使医生可以摆脱计算 机的键盘,直接用声笔通过“莱单”与计算机对话，操作十分简便. 两类程序分别用 Z80 汇编 语言和 FORTRAN 高级语言编写, 允许操作者反复补测、修改、删除及列表等, 当测量结束时,

本文 1984 年 4 月 15 日收到. 
计算机在几十杪内便算出所需的 60 项参数,并打印出一份完整的报告(图 1 $)^{(1-2 .}$.

\section{二、结 果}

1. 探查和测量方法的标准化

我们对心前区探查基本采用 Feigenb$\operatorname{sum}^{[3]}$ 和 Segal ${ }^{[4]}$ 等提出的声束贯穿部位的分 区法:

（1）心底波型的定位，即一般公认探查 4 区, 以显示主动脉解回声为重要标志.

(2) 二尖䅸测量的定位即为 3 区，选择 探头垂直位, 能完整显示二尖㲔前叶及其振 幅最大的波型为准,这时后叶尚未显示,室间 隔左室面力求显示清晰, 而后方为左房后壁.

(3) 在标准 2 区测量左室短轴径的特点

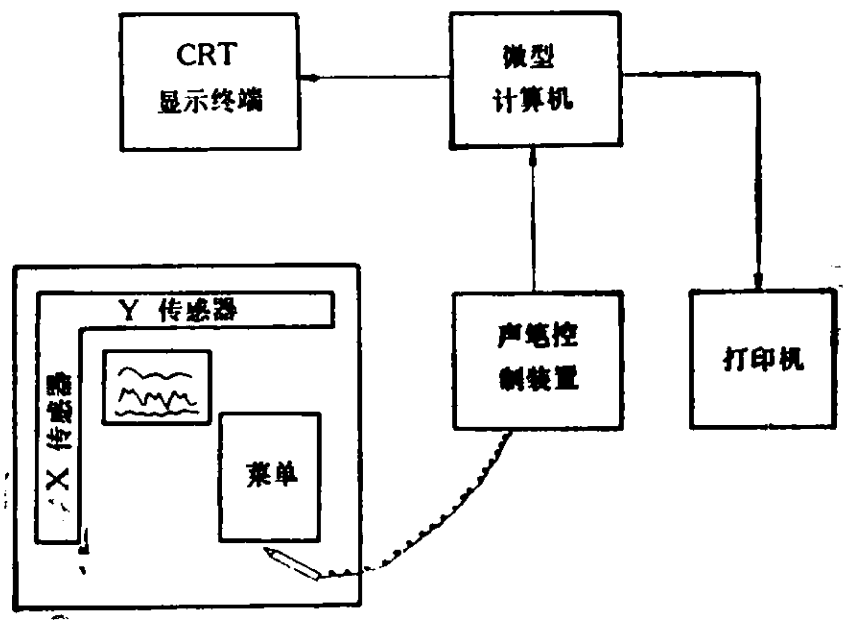

图 1 声笔数字化器——计筆机 自动分析系统示意图 是显示二尖辩前后两叶尖部或腱索,收缩期后壁较舒张期明显增厚; 后壁舒张期运动速度较收 缩期快; 室间隔左室面与左室后壁心内膜面,显示典型的反向运动.

97 例的 UCG 应用声笔数字化器一一计算机分析所得的数据, 选择其中五项, 基本与张 武 $^{[5]}$, 简文豪、任建方 ${ }^{(6)}$ 等作者报道是一致的 (表 1 ).

表 1 正常人 UCG 部分指标值的比较 ( $\mathrm{X}_{ \pm} \mathrm{S}$ 豪米)

\begin{tabular}{|c|c|c|c|c|c|c|}
\hline & & $\begin{array}{c}\text { 闻 恽 等 } \\
\text { (1975) }\end{array}$ & $\begin{array}{l}\text { 张 武等 } \\
(1977)\end{array}$ & $\begin{array}{l}\text { 简文毫等 } \\
\text { (1977) }\end{array}$ & $\underset{(1980)}{\text { 㶵 }}$ & (1983) \\
\hline 例 数 & $\begin{array}{l}\text { 男 } \\
\text { 女 }\end{array}$ & $\begin{array}{l}76 \\
38\end{array}$ & $\begin{array}{l}65-69 \\
51-55\end{array}$ & $\begin{array}{l}30 \\
30\end{array}$ & $\begin{array}{l}30-32 \\
11-12\end{array}$ & $\begin{array}{l}55 \\
42\end{array}$ \\
\hline \multicolumn{2}{|c|}{ 年秢 } & $18-66$ & $16-64$ & $45-66$ & $45-61$ & $19-59$ \\
\hline 主动脉径 & 男 & $\begin{array}{l}28.4 \pm 4.2 \\
24.7 \pm 3.0\end{array}$ & $\begin{array}{l}27.6 \pm 2.9 \\
23.8\end{array}$ & $\begin{array}{l}31.1 \pm 4.6 \\
26.3 \pm 2.8\end{array}$ & $\begin{array}{l}29.9 \pm 2.0 \\
27.5 \pm 2.3\end{array}$ & $\begin{array}{l}28.7 \pm 2.8 \\
25.6 \pm 2.9\end{array}$ \\
\hline 左室舒张期内径 & 男 & $\begin{array}{l}73.0 \pm 7.8 \\
69.6 \pm 7.1\end{array}$ & $\begin{array}{l}46.4 \pm 3.7 \\
41.8 \pm 3.3\end{array}$ & $\begin{array}{l}45.3 \pm 1.0 \\
44.3 \pm 3.5\end{array}$ & $\begin{array}{l}44.2 \pm 3.5 \\
41.9 \pm 2.6\end{array}$ & $\begin{array}{l}49.9 \pm 3.5 \\
45.4 \pm 4.1\end{array}$ \\
\hline 左室收缩期内经 & 男 & $\begin{array}{l}63.7 \pm 8.5 \\
60.1 \pm 9.4\end{array}$ & $\begin{array}{l}29.7 \pm 4.1 \\
27.3 \pm 3.7\end{array}$ & $\begin{array}{l}28.9 \pm 2.7 \\
26.9 \pm 3.6\end{array}$ & $\begin{array}{l}28.1 \pm 3.3 \\
28.4 \pm 2.4\end{array}$ & $\begin{array}{l}34.2 \pm 3.9 \\
29.8 \pm 3.4\end{array}$ \\
\hline 左公后壁舒张期厚度 & $\begin{array}{l}\text { 男 } \\
\text { 女 }\end{array}$ & $\begin{array}{l}9.5 \pm 2.1 \\
9.4 \pm 0.6\end{array}$ & $\begin{array}{l}9.3 \\
8.2\end{array}$ & $\begin{array}{l}6.1 \pm 0.5 \\
6.4 \pm 1.0\end{array}$ & $\begin{array}{l}9.7 \pm 0.8 \\
9.5 \pm 0.5\end{array}$ & $\begin{array}{l}9.1 \pm 1.2 \\
9.0 \pm 1.2\end{array}$ \\
\hline 左房前后径 & 男 & $\begin{array}{l}28.4 \pm 4.7 \\
27.8 \pm 3.4\end{array}$ & $\begin{array}{l}28.3 \pm 3.5 \\
26.7 \pm 2.9\end{array}$ & $\begin{array}{l}33.1 \pm 5.5 \\
29.7 \pm 3.7\end{array}$ & $\begin{array}{l}30.2 \pm 2.2 \\
28.7 \pm 3.0\end{array}$ & $\begin{array}{l}30.7 \pm 3.4 \\
28.7 \pm 3.0\end{array}$ \\
\hline
\end{tabular}

2.97 名健康者的 UCG，用手工方法测量 60 个参数是相当费时间的，一般测 1 例需 40 分 钟才能完成. 因此目前临床上仅选用 10 多个参数作为诊断依据, 远远不能发挥 UCG 的倌息 和其功能. 采用声笔数字化器——微型计算机半自动法（简称半自动法）测量 UCG, 1 分钟 内即可测量完毕，经微型计算机自动运算后，2-3 分钟即可打印出一份报告,就时间来看，提 高工作效率约 10 倍以上. 用本法测得的结果,参数增多,明显提高了 UCG 对科研和辅助攼床 的价值 (另文发表). 选择其中26项参数, 应用手工法和半自动法进行比较, 经统计学处理, 均 
表 2 半自动法讧 UCG 部分参数与手工测量比较 $(\mathrm{X} \pm \mathrm{SDmm})$

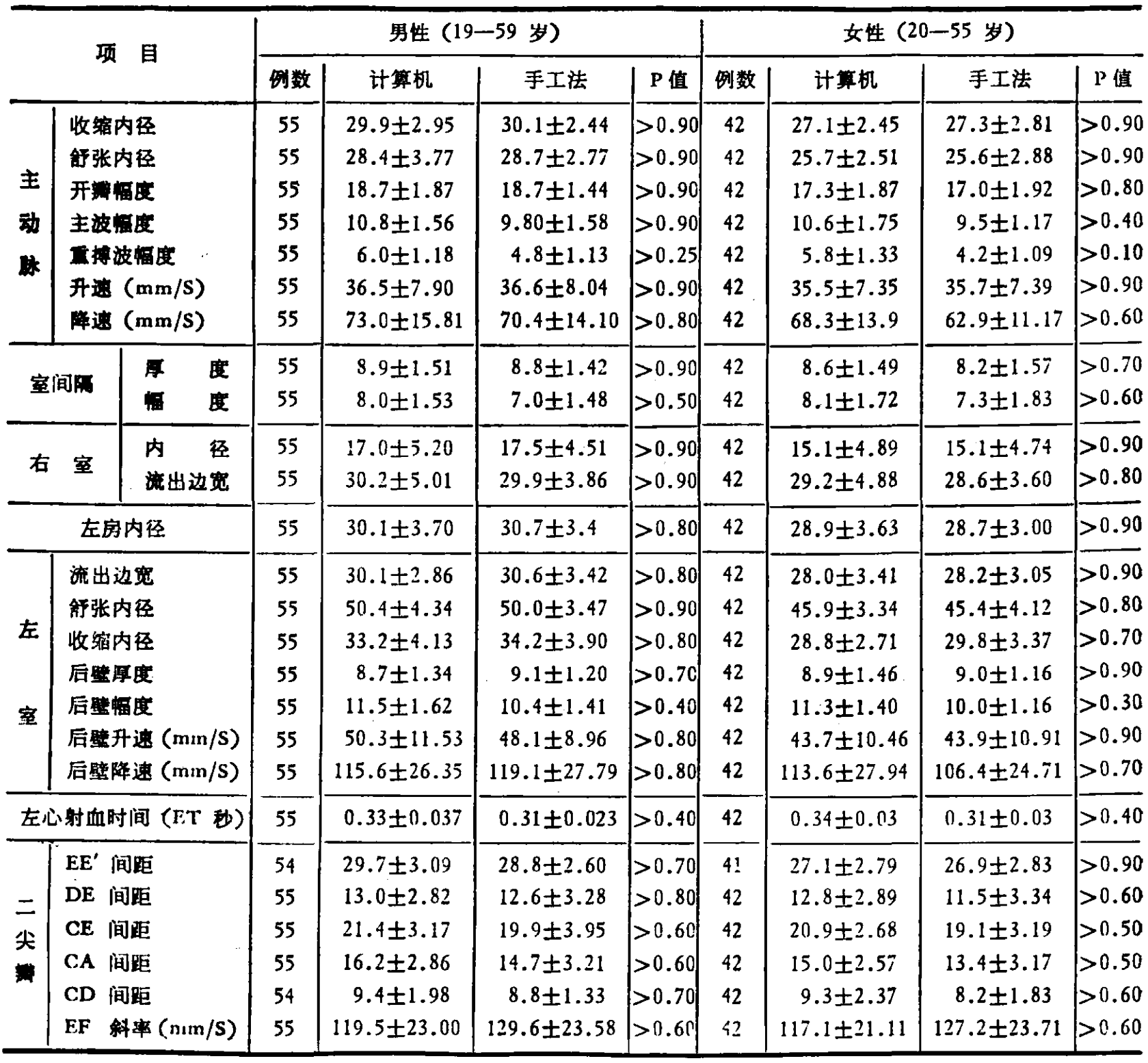

无明显差异 (表 2).

除上述“点”方式测定的 57 个项目外,还有 3 个“线”方式的测定项目,运用“线”测量方法， 可直接定量计算 M 型 UCG 二尖瓣开放时辦膜口的面积 (另文报道)。因此，应用本法可以解 决计算繁琐和用手工方法测量难以取得的数据,获得更多诊断和科研需要的信息。

3. 心肚功能测定对心功能评定、药物治疗效果及判断病情食后有重要的意义. 除应用心 导管及心血管造影等损伤性检查方法外，还有无损伤的检查法如心脏收缩时间间期、心尖搏动 图、左心室阻抗微分图、人体胸阻抗图等,都是测定心功能的方法. 自从 1967 年 Feigenbaum 首 先应用 UCG 测定左心室排血量, 以后有.John 等用 UCG 作左心室周径向心缩短速率的研究, Donford 等作了主动脉返流量及二尖䅸血流量的测定．二尖瓣开放及关闭速度的测定亦有不 少报道. 由此可见,应用 UCG 测定左心功能, 可作反复、动态的定量检查, 对临床又提供了一 个无创伤性、简单易行的辅助诊断工具. 目前左心室排血量的测定原理是：假设左心室是一 个椭圆形或立方形的几何形体, UCG 可测出左心室舒、缩内径及房室环幅度,将测定值代入公 式,可算出左心室舒张及收缩时的体积，两者之差即为左心室排血量. 测定方法有楉圆形体积 
法,立方形体积法,迴归方程法，主动脉、二尖房室环计算法等。我们为了简化,选用立方 体积法将手工法与半自动化法所测结果进行比较，结果表明,两组间左心室每搏量、左心室缩 末、舒末容积、射血分值 EF、左心室小轴缩短率 FS 及左心室周径向心缩短率等,经统计学处理 后均无显著差异 (表 3)。半自动法又能明显提高诊断速度, 使临床医生能更及时得到左心室 排血量的结果,便于观察疗效和愈后.

表 397 例左室功能半自动化分析与手工测量的比较

\begin{tabular}{|c|c|c|c|c|c|c|}
\hline \multirow{2}{*}{ 项 目 } & \multicolumn{3}{|c|}{ 男 性 (55) } & \multicolumn{3}{|c|}{ 女性 (42) } \\
\hline & 类 别 & $\overrightarrow{\mathbf{x}} \pm S D$ & P 值 & 类 别 & $\overline{\mathbf{x}} \pm \mathrm{SD}$ & P 值 \\
\hline 左心每搏量 & 手杢 & $\begin{array}{l}101 \pm 14.0 \\
109 \pm 18.5\end{array}$ & $>0.60$ & 手贡 & $\begin{array}{r}97 \pm 15.5 \\
102 \pm 17.1\end{array}$ & $>0.70$ \\
\hline $\begin{array}{c}\text { 左心每分搏出量 } \\
(\mathrm{ml})\end{array}$ & 手苸算机 & $\begin{array}{l}6779 \pm 1236 \\
7311 \pm 1434\end{array}$ & $>0.60$ & 手 计算机 & $\begin{array}{l}6898 \pm 1369 \\
7288 \pm 1443\end{array}$ & $>0.70$ \\
\hline $\begin{array}{c}\text { 左心缩末容积 } \\
(\mathrm{ml})\end{array}$ & 手 $\frac{I}{\text { 计算机 }}$ & $\begin{array}{l}41 \pm 18.2 \\
36 \pm 19.2\end{array}$ & $>0.80$ & 手杢 & $\begin{array}{l}20 \pm 15.6 \\
16 \pm 12.5\end{array}$ & $>0.70$ \\
\hline $\begin{array}{c}\text { 左心舒末容积 } \\
(\mathrm{ml})\end{array}$ & 手 $\frac{T}{\text { 计算机 }}$ & $\begin{array}{l}142 \pm 20.5 \\
145 \pm 26.2\end{array}$ & $>0.90$ & 手甹 & $\begin{array}{l}117 \pm 23.5 \\
118 \pm 19.7\end{array}$ & $>0.90$ \\
\hline$\underset{\mathrm{EF}}{\text { 射血分值 }}$ & 手 & $\begin{array}{l}0.717 \pm 0.105 \\
0.759 \pm 0.108\end{array}$ & $>0.60$ & 手 & $\begin{array}{l}0.841 \pm 0.102 \\
0.874 \pm 0.092\end{array}$ & $>0.70$ \\
\hline $\begin{array}{c}\text { 左心小轴缩短率 } \\
\text { FS }(\%))\end{array}$ & 手 & $\begin{array}{l}31.65 \pm 5.19 \\
34.21 \pm 5.84\end{array}$ & $>0.60$ & 手甹 & $\begin{array}{l}34.23 \pm 6.79 \\
37.07 \pm 5.33\end{array}$ & $>0.60$ \\
\hline $\begin{array}{l}\text { 左室周径向心缩 } \\
\text { 短率AD/SEC }\end{array}$ & 手 计算机 & $\begin{array}{l}1.031 \pm 0.198 \\
1.049 \pm 0.222\end{array}$ & $>0.90$ & 手 $\frac{I}{\text { 计算狄 }}$ & $\begin{array}{l}1.132 \pm 0.186 \\
1.114 \pm 0.178\end{array}$ & $>0.90$ \\
\hline
\end{tabular}

4. 应用电子计算机分析 UCG 在国内尚未见报道. 自 70 年代以来, 微处理机迅速发展, 国外已将它用于 M 型 UCG 数值的分析, 初步已显示出它的优越性, 促进了心血管疾病的研究 和诊断. 要实现 UCG 的计算机辅助分析, 首先要把图象的各种信息,转换为数字舅, 然后由 计算机进行处理. 目前这方面的技术包括:（1）实时数字化；(2) 直接数字化；(3)间接数字 化. 前两种方法都需要有较高级的计算机和相当复杂的计算方法, 取得有用的信息, 不仅代 价昂贵，而且对参与这项工作者的技术条件要求高. 间接数字化要求工作人员条件较低，仪器 设备也较低廉,只要一台声笔数字化系统和微型计算机,甚至有一个可编程序的袖珍计算器即 能满足，而且不需要复杂的处理程序. 本系统软件的设计考虑到使用者可能是对计算机不很 熟悉的内科医生,故使之达到操作简化、方便、测量项目多、速度快. 线测量的各种结果是手工 无法进行的,这是该系统的突出优点，随着应用更普遍、更深人，这一优点将更会显示其优越 性. 因此,结合我国目前的国情,本法易于被一般医院和实验室接受并推广.

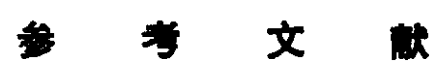

[1] Krcbs, N., Computer processing of one-and-two-dimensional echocardiograms, Biomedizinische Technik, 24(1979), 242.

[ 2 ] Feigenbaum, H., Clinical application of echocarechocardiograms, J. of Clinical Ultrasound, 4(1976), 173

[ 3 ] Pai, A. L., Digital computer analysis of M-scan diography, Progr. Cardiovasc. Dis., 14(1972), 531.

[ 4 ] Segal, B. L. et al., Echocardiography current concepto and clinical application, Amer. J. Med., 57(1974), 267.

[5] 张武, 124例正常超声心动图的测量与分析, 中华医学杂志, 57(1977), 287.

[6]任基方,正常人招声心动图的评价,北铁医刊, 5(1980),1. 